\title{
Recombinant attenuated Salmonella typhimurium carrying a plasmid co-expressing ENDO-VEGI151 and survivin siRNA inhibits the growth of breast cancer in vivo
}

\author{
ZHE LI $^{1 *}$, PEI-HAO YIN ${ }^{3 *}$, SHENG-SHENG YANG ${ }^{4}$, QIAN-YU LI ${ }^{2}$, TAO CHANG $^{1}$, \\ LIN FANG $^{1}$, LIN-XIANG SHI $^{1}$ and GUO-EN FANG ${ }^{5}$
}

Departments of ${ }^{1}$ Thyroid and Breast Surgery, and ${ }^{2}$ Pathology, The Shanghai Tenth People's Hospital, Tong Ji University, Shanghai 200072; ${ }^{3}$ Department of General Surgery, Putuo Hospital, Shanghai University of Traditional Chinese Medicine, Shanghai 200062; Departments of ${ }^{4}$ Biochemistry and Molecular Biology, and ${ }^{5}$ General Surgery of Changhai Hospital, Second Military Medical University, Shanghai 200433, P.R. China

Received October 20,2012; Accepted January 22, 2013

DOI: $10.3892 / \mathrm{mmr} .2013 .1308$

\begin{abstract}
The aim of this study was to investigate the antitumor effect of a plasmid co-expressing ENDO-VEGI151 and survivin siRNA on breast cancer in nude mice, and to explore the feasibility of attenuated Salmonella typhimurium (S. typhimurium) as a delivery vector for cancer gene therapy in vivo. Three recombinant expression plasmids pENDO-VEGI151 (pEV), pSurvivin-siRNA (psi-survivin) and co-expressing plasmid pENDO-VEGI151/survivin-siRNA ( $\mathrm{pEV} / \mathrm{si}$-survivin), were transferred into the attenuated S. typhimurium strain SL7207, respectively. MDA-MB-231 cells were infected with these recombinants in vitro, and the expression of ENDO-VEGI151 and survivin was detected. In order to detect $S$. typhimurium distribution and gene delivery efficiency in vivo, the plasmid pEGFP-N1 which encodes green fluorescent protein was transferred into SL7207, and the recombinant known as SL-pEGFP was orally administered to tumor-bearing nude mice. The gene transfer efficiency, distribution and survival time of the SL-pEGFP in vivo were evaluated by detection of GFP fluorescence. SL-pEGFP not only infected the cancer cells effectively, but also allowed the survival and expression of specific genes mainly in the xenografts of nude mice. To further identify the anticancer effects of these recombinants in vivo, mice burdened with xenografts were randomly divided into 6 groups, which were subjected to intragastric administration of vehicle, SL7207, SL-pcDNA3.1, SL-pEV, SL-psi-survivin and SL-pEV/si-survivin, respectively.
\end{abstract}

Correspondence to: Dr Zhe Li, Department of Thyroid and Breast Surgery, The Shanghai Tenth People's Hospital, Tong Ji University, 301 Shanghai Yanchangzhong Road, Shanghai 200072, P.R. China E-mail: lizhe@medmail.com.cn

${ }^{*}$ Contributed equally

Key words: breast cancer, survivin, anti-angiogenensis, attenuated Salmonella typhimurium, gene therapy
Eight weeks after implantation, tumor size, weight, inhibition rate, intratumoral microvessel density (MVD), apoptotic index (AI), ENDO-VEGI151 and survivin expression were evaluated. Compared with the SL-pEV or SL-psi-survivin-treated groups, the growth of tumors was significantly reduced in the SL-pEV/si-survivin group with an inhibition rate of 90.28 vs. 69.12 and $65.61 \%$, respectively. MVD and the expression of survivin were decreased significantly in the SL-pEV/ si-survivin-treated group, while AI increased significantly in the SL-pEV/si-survivin-treated group. These results indicated that attenuated $S$. typhimurium carrying the dual function plasmid pEV/si-survivin cannot only be specifically enriched in the tumor tissue, but also showed a synergistic antitumor effect in vivo.

\section{Introduction}

The incidence of breast cancer has increased annually, and is a major threat to women's health. With the application of new chemotherapy drugs and molecular-targeted therapy, the treatment of breast cancer has made marked progress, but there remains no effective treatment for metastatic breast cancer, and its mortality has not significantly reduced $(1,2)$.

Gene therapy, considered as a promising alternative treatment, has received increasing attention and has shown good application prospects (3). Tumors are composed of cancer and endothelial cells, which are interdependent and have a mutually reinforcing vicious cycle $(4,5)$. Therefore, the best treatment strategy is to kill cancer cells and destroy the cancer cell microenvironment, which includes neovascular endothelial cells, infiltrated immune cells and mesenchymal cells. In this study, we successfully constructed a bifunctional plasmid expressing ENDO-VEGI151 and survivin siRNA, and confirmed that it not only promotes tumor cell apoptosis, but also has anti-angiogenic effects. On the basis of these bifunctional activities (6), attenuated Salmonella typhimurium (S. typhimurium) was selected to carry the plasmid, and a human breast cancer model was established in nude mice to further evaluate the efficacy of this new approach in vivo. The 
purpose of this study was to evaluate comprehensive, effective, convenient (oral bacteria) and cheaper new approaches for breast cancer gene therapy.

\section{Materials and methods}

Reagents. Plasmids pENDO-VEGI151 (pEV), pENDOVEGI151/survivin-shRNA(pEV/si-survivin),pSurvivin-siRNA (psi-survivin) and pcDNA3.1 were constructed and preserved by our laboratory. The plasmid pEGFPN1, mouse Salmonella LB5000 and attenuated S. typhimurium SL7207 were kindly provided by Professor Zhao Ping from the Department of Microbiology, Second Military Medical University, China. Human breast cancer cells MDA-MB-231 were purchased from the cell bank of the Chinese Academy (Shanghai, China). Rabbit anti-human survivin polyclonal antibody was purchased from Santa Cruz Biotechnology, Inc. (Santa Cruz, CA, USA), rabbit anti-human VEGI151 polyclonal antibody was kindly provided by Dr Zhang Min (Department of Microbiology, Second Military Medical University), and the mouse antihuman factor VIII-related antigen monoclonal antibody was purchased from Dako (Carpinteria, CA, USA). $\beta$-actin antibody was purchased from Sigma (St. Louis, MO, USA). The apoptosis detection kit was purchased from GenScript (Piscataway, NJ, USA). An ultra-sensitive, SP immunohistochemistry kit was purchased from Mai-xin Bio (Fuzhou, China). Healthy, purebred, SPF, 4-6-week-old, female Balb/c nude mice were purchased from the Experimental Animal Center of Second Military Medical University.

Preparation of recombinant bacteria. Plasmids $\mathrm{pEV} /$ si-survivin, $\mathrm{pEV}$, psi-survivin, pcDNA3.1 and pEGFPN1 were transformed into calcium chloride-treated murine S. typhimurium LB5000 cells, plasmids were extracted using the Gene Pulser (Bio-Rad, Hercules, CA, USA) electroporation instrument. We obtained 5 types of recombinant Salmonella known as SL-pEV/si-survivin, SL-pEV, SL-psi-survivin, SL-pcDNA3.1 and SL-pEGFP. Salmonella SL7207 cells were attenuated with $10 \%$ glycerol.

Recombinant bacterial in vitro infection and identification of therapeutic gene expression. Breast cancer cells MDA-MB-231 were inoculated into 24-well plates, grown to $80 \%$ confluence, washed with phosphate-buffered saline (PBS) four times, and SL-pEV/si-survivin, SL-pEV, SL-psi-survivin, SL-pcDNA3.1, or untransformed plasmid empty bacteria SL7207 [ $1 \times 10^{6}$ colony forming units (CFU)] were added into the recombinant bacteria, respectively. One hour later at room temperature, PBS containing $50 \mu \mathrm{g} / \mathrm{ml}$ kanamycin was used for washing 3 times, then cells were incubated with culture medium RPMI-1640 containing the same concentration of kanamycin (to kill extracellular bacteria) for $4 \mathrm{~h}$. A final concentration of $10 \mu \mathrm{g} / \mathrm{ml}$ of tetracycline (inhibition of intracellular bacteria proliferation) was then added, cells were incubated for $48 \mathrm{~h}$, then collected and lysed. Real-time fluorescence quantitative PCR and western blot analysis were performed in order to detect the expression of ENDO-VEGI151 and survivin.

Establishment of the human breast cancer xenograft model and evaluation of bacterial distribution in animals. Breast cancer cells MDA-MB-231 growing in the logarithmic phase were collected and inoculated into nude mice subcutaneously. Tumors were detected after 10 days. Tumor-bearing nude mice were divided into 2 groups $(\mathrm{n}=6)$ and administered with $1 \times 10^{9}$ colony forming $\mathrm{U} / \mathrm{ml}\left(\mathrm{cfu} / \mathrm{ml}^{-1}\right.$ ) of SL7207 (control group) and $0.1 \mathrm{ml}\left(\sim 10^{8} \mathrm{cfu}\right)$ SL-pEGFP (experimental group). Tumor-bearing nude mice were sacrificed after inoculation at 24 and 48 h, 5, 10, 20 and 35 days, respectively. Frozen sections $(12 \mu \mathrm{m})$ obtained from liver, spleen and tumor tissues were analyzed with confocal microscopy (Leica TCS NT, Wetzlar, Germany). Green fluorescent protein (GFP) was observed and calculated to evaluate the distribution and survival time of SL-pEGFP in vivo. The use of nude mice for this study was approved by the Institutional Animal Care and Use Committee at the Second Military Medical University, Shanghai, China.

Oral treatment of the tumor-bearing nude mice with recombinant bacteria carrying a bifunctional expression plasmid. Breast cancer cells were transplanted into the nude mice model until tumors grew to $100 \mathrm{~mm}^{3}$, then the mice were randomly divided into 6 groups: PBS, SL7207 empty bacteria, SL-pcDNA3.1, SL-pEV, SL-psi-survivin and SL-pEV/ si-survivin $(\mathrm{n}=5)$. Each nude mouse was administered 5\% $\mathrm{NaHCO}_{3} 0.2 \mathrm{ml}$ by oral gavage, then given the corresponding recombinant $S$. typhimurium (dosage $1 \times 10^{8} \mathrm{cfu} / 0.2 \mathrm{ml}$ ) by oral gavage after $30 \mathrm{~min}$. The control group was administered PBS by gavage. Mice were inoculated 3 times, at an interval of 7 days. The longest diameter (a) and shortest diameter (b) of the tumors were measured weekly with vernier calipers and the tumor volume was calculated according to the formula: $\mathrm{V}=\pi \mathrm{ab}^{2} / 6$. Mice were sacrificed at 8 weeks and the tumor was separated from the normal tissues in order to calculate the tumor volume. Tumors were weighed after cleaning with saline. After fixation with $15 \%$ neutral buffered formalin, tumors were evaluated with conventional biopsy and hematoxylin-eosin staining. FVIII factor, survivin and ENDO-VEGF151 were detected by immunohistochemical staining and apoptosis analysis was determined by TUNEL staining. Microvessel density (MVD) was counted under x200 objective light microscopy; factor VIII antibody was used to show the vascular numbers, and the results of the average number of blood vessels in five fields were evaluated. The apoptotic index (AI) was evaluated with $\mathrm{x} 400$ objective light microscopy. Five tumor areas were selected randomly and the proportion of apoptosis-positive cells was calculated in all cancer cells and the mean value was calculated.

Statistical analysis. SPSS 13.0 statistical software was used for analysis of variance. $\mathrm{P}<0.05$ was considered to indicate a statistically significant difference. The mapping was achieved using Excel (Microsoft).

\section{Results}

The efficiency of the recombinant S. typhimurium infection and evaluation of expression. After 48-h infection of MDA-MB-231 cells with the recombinant bacteria, real-time PCR results revealed that infection with recombinant strain SL-psi-survivin and SL-pEV/si-survivin reduced survivin mRNA levels by 2.28 and 2.57 times, respectively, while the 

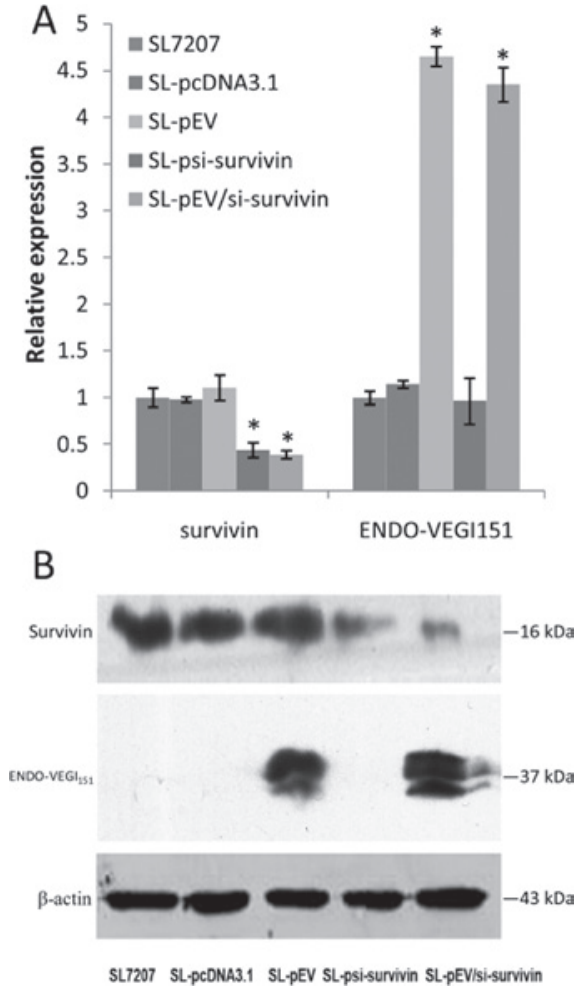

Figure 1. Expression of survivin and ENDO-VEGI151 were identified by (A) RT-PCR and (B) western blotting. After infection with different recombinant Salmonella typhimurium (S. typhimurium) strains, tumor cells were collected and lysed for protein and RNA extraction. The histogram shows the means \pm SD of triplicate RT-PCR determination. ${ }^{*} \mathrm{P}<0.05$, compared with the SL7207 and SL-pcDNA3.1 groups.
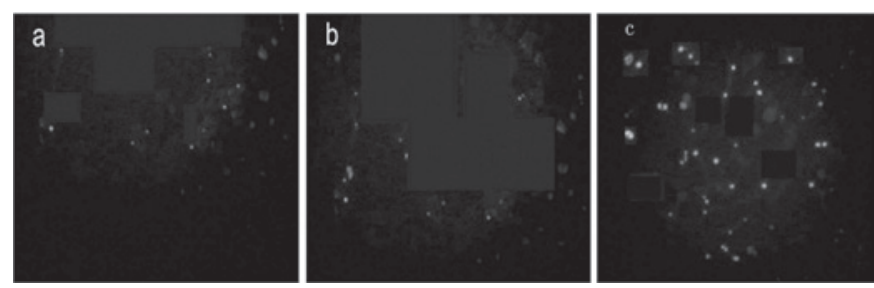

Figure 2. Characterization of SL-pEGFPN1-mediated gene transfection to cells after oral treatment in nude mice. Expression of naturally emitting green fluorescent protein (GFP) was determined by confocal imaging in (A) spleen, (B) liver and (C) tumor cells from nude mice receiving the Salmonella typhimurium (S. typhimurium) SL7207 strain carrying the expression vector SL-pEGFPN1, which expresses GFP under the control of a eukaryotic promoter.

corresponding inhibition rates were 56 and $61 \%$, respectively $(\mathrm{P}<0.01)$. Survivin mRNA levels in the recombinant strain SL-pEV and SL-pcDNA3.1-infected cells were similar to that of empty bacteria SL7207-infected cells. Infection with recombinant strain SL-pEV and SL-pEV/si-survivin increased the ENDO-VEGI151 mRNA level by 4.6 and 4.3 times $(\mathrm{P}<0.01)$, while the ENDO-VEGI151 mRNA level in cells infected with the recombinant strain SL-psi-survivin and SL-pcDNA3.1 was similar to that of empty bacteria SL7207-infected cells (Fig. 1A). Western blot analysis further confirmed at the protein level that SL7207-pEV/Si-survivin infection can inhibit the expression of survivin effectively while expressing ENDO-VEGI151 fusion protein at the same time (Fig. 1B).

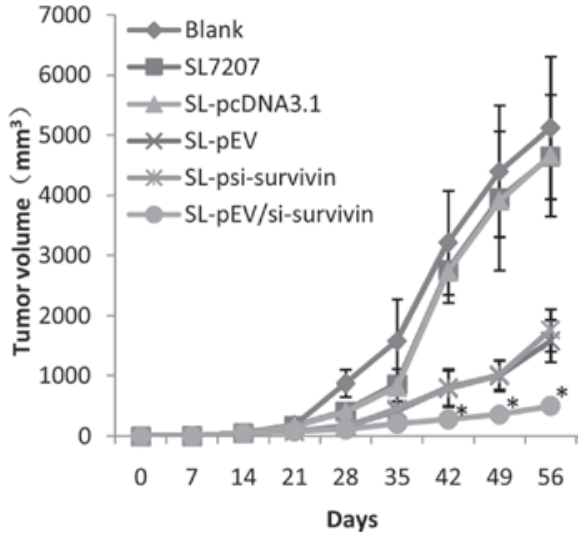

Figure 3. Xenograft growth curves following inoculation with various recombinant attenuated Salmonella typhimurium (S. typhimurium) strains. Data are presented as the means $\pm \mathrm{SD}(\mathrm{n}=5),{ }^{*} \mathrm{P}<0.05$, compared with other groups.

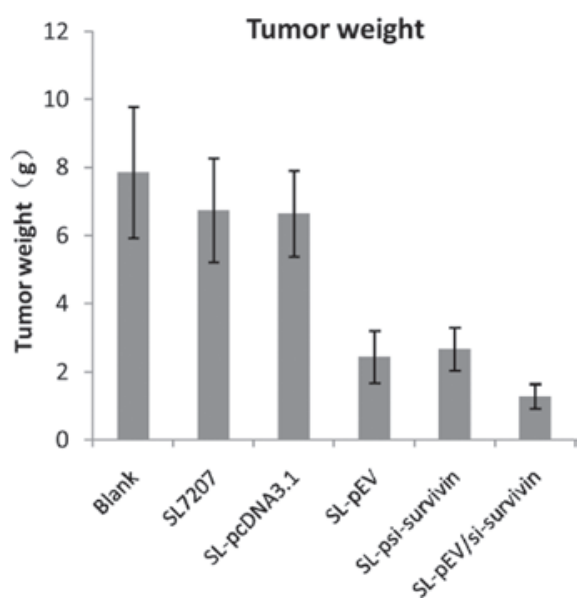

Figure 4. Weight of xenografts following treatment with various Salmonella recombinants. Data are presented as the means $\pm \operatorname{SD}(n=5)$.

Recombinant bacterial distribution and survival time in nude mice. GFP expression was detected in a few organs at $24 \mathrm{~h}$, while marked GFP signals were detected in tumors at $48 \mathrm{~h}$. Spotty and sparse signals (Fig. 2) were observed in liver and spleen tissues. GFP expression peaked at 5 days, with the expression almost disappearing after 10 days. GFP expression in tumor tissues was still visible at 35 days, while no fluorescent signal was detected in the control group.

The effect of recombinant bacterial treatment on breast cancer cell tumor growth in nude mice. Following inoculation with MDA-MB-231 cells for 7 days, breast tumors were detected in nude mice. The tumor growth curve in nude mice after breast cancer cell inoculation is shown in Fig. 3. At day 15 after cancer cell inoculation, vernier calipers were used to measure tumor volume in order to monitor the tumor growth. The volume of tumors from PBS, SL7207 empty bacteria, SL-pcDNA3.1, SL-pEV, SL-psi-survivin and SL-pEV/ si-survivin groups was measured from the 21st day (35 days) of the start of treatment (Fig. 4). The transplanted tumors in the SL-pEV, SL-psi-survivin and SL-pEV/si-survivin groups grew slower than the blank group with a statisti- 
Table I. Nude mice tumor volume, weight and inhibition rate.

\begin{tabular}{lcccc}
\hline & $\begin{array}{c}\text { Tumor volume } \\
\text { Groups }\end{array}$ & $\begin{array}{c}\text { Tumor weight } \\
(\mathrm{g}, \text { mean } \pm \mathrm{SD})\end{array}$ & Volume $(\%)$ & Weight $(\%)$ \\
\cline { 4 - 5 } Vehicle control & $5119.64 \pm 1180.44$ & $7.86 \pm 1.93$ & - & - \\
SL7207 & $4644.69 \pm 1007.81$ & $6.74 \pm 1.53$ & 9.27 & 14.25 \\
SL-pcDNA3.1 & $4661.68 \pm 805.22$ & $6.64 \pm 1.26$ & 8.95 & 15.52 \\
SL-pEV & $1580.87 \pm 349.28^{\mathrm{a}}$ & $2.44 \pm 0.77^{\mathrm{a}}$ & $69.12^{\mathrm{a}}$ & $68.96^{\mathrm{a}}$ \\
SL-psi-survivin & $1760.85 \pm 350.71^{\mathrm{a}}$ & $2.68 \pm 0.63^{\mathrm{a}}$ & $65.61^{\mathrm{a}}$ & $66.16^{\mathrm{a}}$ \\
SL-pEV/si-survivin & $497.48 \pm 107.37^{\mathrm{a}, \mathrm{b}}$ & $1.28 \pm 0.36^{\mathrm{a}, \mathrm{b}}$ & $90.28^{\mathrm{a}, \mathrm{b}}$ & $83.72^{\mathrm{a}, \mathrm{b}}$ \\
\hline
\end{tabular}

${ }^{\mathrm{a} P}<0.05$ compared with SL7207, SL-pcDNA3.1 and vehicle control groups. ${ }^{\mathrm{b}} \mathrm{P}<0.05$ compared with SL-pENDO-VEGI151 (pEV) and SL-psisurvivin groups.

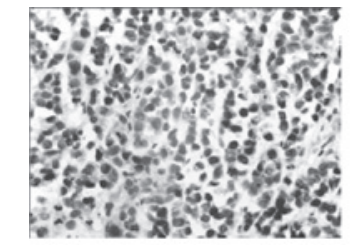

BLANK

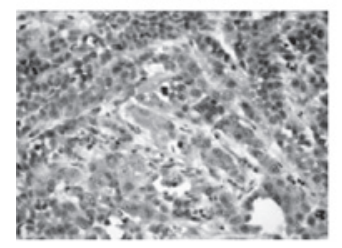

SL-pEV

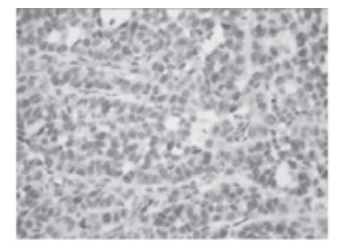

SL7207

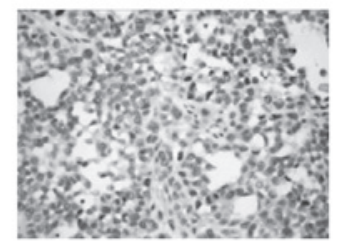

SL-psi-survivin

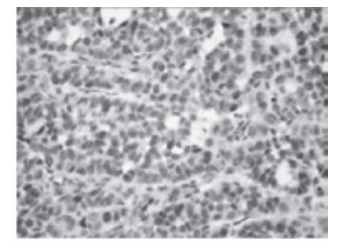

SL-pcDNA3.1

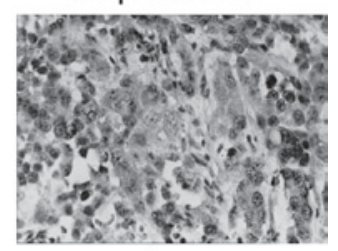

SL-pEV/si-survivin

Figure 5. Immunohistochemical analysis of ENDO-VEGI151 expression in MDA-MB-231 xenografts treated with variant Salmonella typhimurium (S. typhimurium) recombinants (x400).

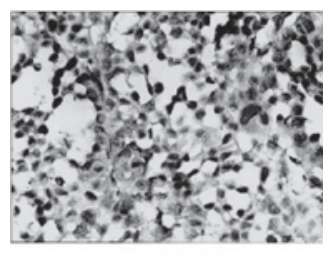

BLANK

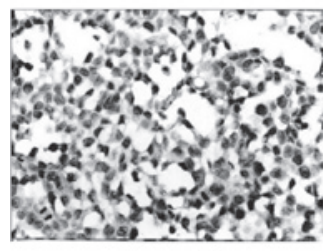

SL-pEV

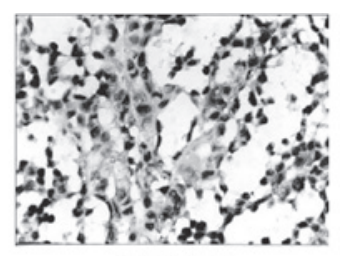

SL7207

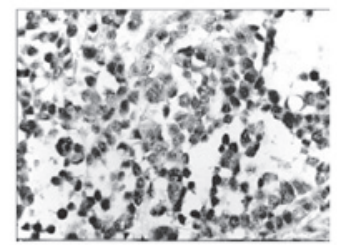

SL-psi-survivin

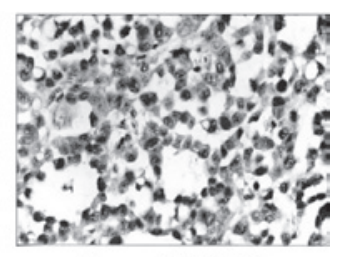

SL-pcDNA3.1

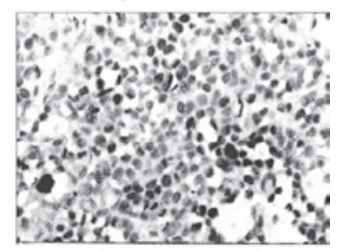

SL-pEV/si-survivin

Figure 6. Immunohistochemical analysis of survivin expression in MDA-MB-231 xenografts treated with variant Salmonella typhimurium (S. typhimurium) recombinants $(\mathrm{x} 400)$.

cally significant difference $(\mathrm{P}<0.05)$. From the 35 th day, the tumor volume of the SL-pEV/si-survivin treatment group was significantly lower than that of the SL-pEV and SL-psisurvivin groups. The difference was more significant at the end of treatment $(\mathrm{P}<0.01)$. Compared with the PBS group, attenuated Salmonella inhibited tumor growth at an early stage with no statistically significant difference $(\mathrm{P}>0.05)$, and its inhibitory effect diminished from the 49th day, at which point the growth rate of the tumor accelerated suddenly. The results show that SL-pEV/si-survivin significantly inhibits tumor growth, while SL7207 and SL-pcDNA3.1 groups have minimal impact on the efficacy (Table I). This shows that 


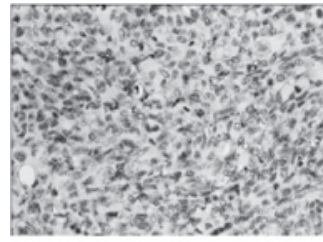

BLANK

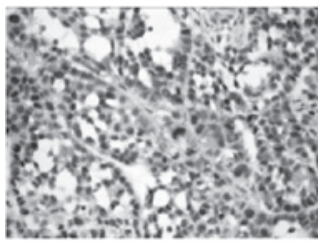

SL-pEV

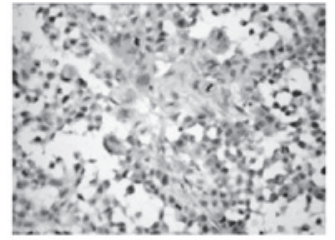

SL7207

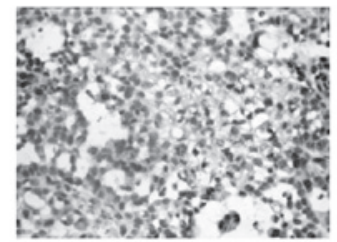

SL-psi-survivin

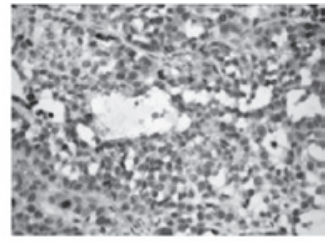

SL-pcDNA3.1

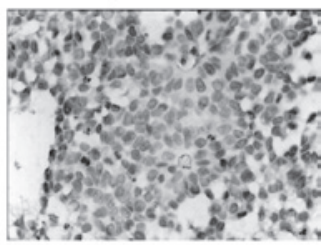

SL-pEV/si-survivin

Figure 7. Microvessel staining of transplanted tumors (x400).

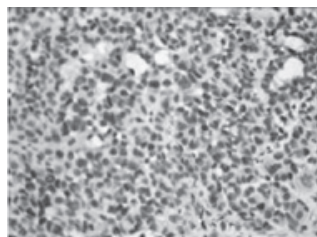

BLANK

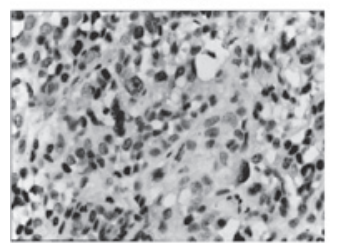

SL-pEV

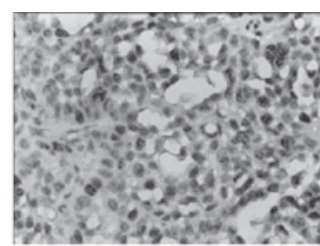

SL7207

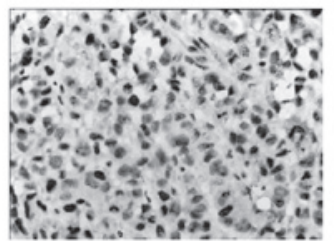

SL-psi-survivin

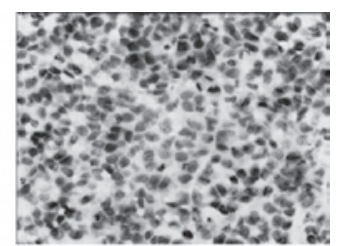

SL-pcDNA3.1

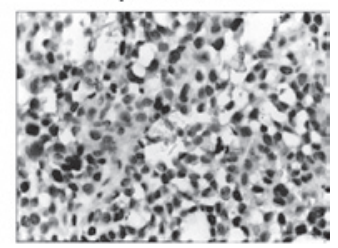

SL-pEV/si-survivin

Figure 8. TUNEL staining of transplanted tumor tissues in nude mice (x400).

the efficacy is not caused by the bacterial carrier, but that the therapeutic genes play a role. The antitumor effects of the SL-pEV/si-survivin, SL-pEV and SL-psi-survivin groups were significantly better than that of the SL-pcDNA3.1 group. The SL-pEV/si-survivin treatment is significantly better than the SL-psi-survivin or SL-pEV groups $(\mathrm{P}<0.01)$. There was no significant difference $(\mathrm{P}>0.05)$ between the SL7207 and SL-pcDNA3.1 groups.

The evaluation of the bifunctional expression plasmid in xenografts. ENDO-VEGI151 protein expression was detected in transplanted tumors from the SL-pEV and SL-pEV/ si-survivin groups. The positive particles were distributed in the cytoplasm and extracellular matrix. Due to the presence of IL3 signal, the fusion protein is secreted into the extracellular matrix in order to execute its function. There was no fusion protein expression in the other groups (Fig. 5). Survivin expression was detected in the tumor tissues of all groups. The positive signal was mainly detected in the cytoplasm of tumor cells with some located within the nucleus. The expression of survivin in transplanted tumors of the SL-psi-survivin and SL-Pev/si-survivin groups was significantly lower than that of the other treatment groups (Fig. 6). This indicates that SL-pEV/si-survivin effectively expressed ENDO-VEGI151 fusion protein in vivo in transplanted tumor tissue while reducing the expression of survivin protein.

Effects of bifunctional expression plasmid treatment on angiogenesis and apoptosis in nude mice. Tumor tissue sections stained with positive FVIII factor signal were brown. Intensive, positive staining of endothelial cells could be observed in the tumor stroma of the control group with uneven microvascular distribution. The dense area of staining was located at the edge of the foci. The blood vessels in the group treated with SL-pEV/si-survivin or SL-pEV were sparse, while the tumor cells grew around the vessels which had 4-6 layers and large nuclei were deeply stained. The tumor cells outside these layers have apoptotic morphology. Large areas of coagulative necrosis appeared around the apoptosis cells (Fig. 7). In the SL-pEV/si-survivin, SL-pEV and SL-psi-survivin treatment groups, tumor cell apoptosis increased significantly with clustered distribution, while the SL7207, SL-pcDNA3.1 and control groups had only a scattered distribution of apoptotic cells (Fig. 8).

MVD in the SL-pEV/si-survivin, SL-pEV and SL-sisurvivin groups was significantly lower than that in the SL-pcDNA3.1, SL7207 and control groups $(\mathrm{P}<0.05)$. AI from the SL-pEV/si-survivin, SL-pEV and SL-si-survivin treatment 
Table II. MVD, AI and survivin expression in tumors of nude mice.

\begin{tabular}{lccc}
\hline Groups & MVD & AI & Survivin \\
\hline Vehicle control & $52.32 \pm 6.48$ & $13.52 \pm 4.85$ & $82.20 \pm 4.46$ \\
SL7207 & $42.44 \pm 3.81$ & $11.84 \pm 3.35$ & $77.59 \pm 5.79$ \\
SL-pcDNA3.1 & $40.28 \pm 4.89$ & $11.73 \pm 2.92$ & $77.64 \pm 5.51$ \\
SL-pEV & $16.52 \pm 6.95^{\mathrm{a}}$ & $49.83 \pm 4.71^{\mathrm{a}}$ & $65.59 \pm 5.52^{\mathrm{a}}$ \\
SL-si-survivin & $28.44 \pm 4.39^{\mathrm{a}}$ & $54.71 \pm 7.63^{\mathrm{a}}$ & $38.03 \pm 5.44^{\mathrm{a}}$ \\
SL-pEV/si-survivin & $13.48 \pm 4.95^{\mathrm{a}, \mathrm{b}}$ & $60.09 \pm 5.89^{\mathrm{a}, \mathrm{b}}$ & $36.46 \pm 5.98^{\mathrm{a}}$ \\
\hline
\end{tabular}

MVD, microvessel density; AI, apoptotic index. ${ }^{a} \mathrm{P}<0.05$, compared with SL-pcDNA3.1, SL7207 and vehicle control groups. ${ }^{\text {b }}<<0.05$ compared with SL-pENDO-VEGI151 (pEV) or the SL-si-survivin groups.

groups increased significantly compared with SL-pcDNA3.1, SL7207 and control groups $(\mathrm{P}<0.05)$. Compared with the two single genes and the control group, MVD reduced significantly in the SL-pEV/si-survivin bifunctional plasmid treatment group with a significant increase in the proportion of apoptotic cells. Survivin expression was significantly decreased (Table II) with a statistically significant difference between the various groups $(\mathrm{P}<0.05)$.

\section{Discussion}

With a deeper understanding of tumor molecular pathology, gene therapy is gaining increasing attention as a cancer treatment, and shows great promise. Currently, cancer gene therapy includes several treatment types: tumor gene antagonists (tumor suppressor gene transfection/tumor gene inactivation) and induction of apoptosis genes (pro-apoptotic gene introduction/inactivation of anti-apoptotic genes), immune genes (cytokine/modified antigen-presenting cell/tumor vaccine), oncolytic viral genes, transduction suicide genes, multidrug resistance genes and anti-angiogenic genes. Tumor vaccines, drug resistance gene and anti-angiogenic gene therapies have achieved promising success in the laboratory and clinical trials have been initiated (7). Tumorigenesis is a complex biological process involving the activation of a variety of oncogenes and the inactivation of tumor suppressor genes and other gene mutations. In addition, tumors are composed of variant cell types; therefore, gene therapy focusing on a single target achieved limited success (8). The synergistic effects of multi-targets using combined gene therapy should in theory be more effective than a single gene target $(9,10)$, and be worthy of further investigations. However, the problem is how to select ideal targets from a large number of candidates.

Abdollahi and Folkman (11) proposed the idea that tumors could be starved through blood vessel blockade and the anti-angiogenic doctrine has persisted for almost 40 years of accumulated research. The central role of angiogenesis in tumor development and its value as a therapeutic target has been recognized since 1971. A large number of retrospective studies have shown that breast cancer is a vascular-dependent disease and angiogenesis is important in breast cancer invasion and metastasis and is an independent prognostic factor. We selected tumor angiogenesis as a target for antiangiogenic therapy and it shows good value and broad application prospects in a number of large randomized trials $(12,13)$. Compared to conventional therapy targeting tumor cells, anti-angiogenic therapy takes the relatively stable genetic traits of vascular endothelial cells as a therapeutic target; vascular endothelial cells possess two high (high targeting specific, high efficiency) and two low ( low adverse reactions, low resistance) properties. Particularly for the resistance of micro-metastases and circulating tumor cells in the patients' body, anti-angiogenic therapy through neovascularization inhibition blocks these micrometastases due to lack of oxygen, and as it maintains the cells at a low mass, it not only notably reduces the metastatic potential, but also makes the cells more susceptible to attack by the immune system and prone to apoptosis. However, antiangiogenic therapy is only a tumor inhibitory treatment; therefore, it cannot completely remove the remaining cancer cells in the patients' body. We hypothesize that combination with direct anticancer therapy is likely to remove tumors completely $(14,15)$. The hypoxic environment induced by anti-angiogenic treatment increases the sensitivity of cancer cells to apoptosis induction therapy $(16,17)$. Therefore, a combination of anti-angiogenesis with induction of apoptosis may achieve the desired therapeutic effect through the two mutually reinforcing synergistic complementary mechanisms.

Based on above assumptions, we used the pre-constructed anti-angiogenesis gene ENDO-VEGI151 and survivin siRNA bifunctional expression plasmid to induce tumor cell apoptosis and inhibit angiogenesis simultaneously. Survivin is known as the strongest anti-apoptotic factor (18), and has a correlation with anti-angiogenesis drug resistance (19). HIF-1 expression is closely related with survivin (20), and so is the proliferation of endothelial cells (21). Thus we selected survivin as the apoptosis induction therapeutic target. In vitro experiments showed that the dual-function plasmid was capable of expressing the ENDO-VEGI151 fusion protein and survivin siRNA in the transfected breast cancer cells MDA-MB-231. In vitro transfection experiments confirmed that the bifunctional expression plasmid effectively inhibits the proliferation of breast cancer and endothelial cells, and also induces apoptosis. It is expected to establish a mutually reinforcing positive feedback antitumor mechanism in the body, which can play cooperative complementary roles. 
There are always safety and efficiency concerns about tumor gene therapy using vector targeting. In addition, the main limitation of the viral vectors (including adenovirus and retrovirus) used currently is that hypoxic phenomena weaken the function of the viral vectors and the expression of target genes in tumors, and certain cells are unlikely to be transfected by the viral vector system, which will mean the tumor has the ability to grow again. Although the solid tumor hypoxia phenomenon is fatal to viral vectors, Salmonella is suitable for a hypoxic environment. Hypoxia promotes Salmonella growth and reproduction (22). Salmonella is an aggressive intracellular bacterium, and the response between it and the host is mediated by the type III secretion mechanism. This mechanism causes the bacterial performance protein transfer into eukaryotic cells and directly expresses a variety of therapeutic proteins. These two features made Salmonella a suitable choice of tumor gene targeting vector. With genetic engineering techniques, we were able to knock out the Salmonella pathogenicity gene to obtain attenuated strains in order to guarantee the safety of the vector (23). The most attractive features of attenuated Salmonella are that it is capable of infecting the tumor cells through vaccination by the mucosal route (oral or nasal) and as a biological carrier its unique remote tumor targeting effect has been demonstrated in the treatment of a variety of tumor models. Attenuated S. typhimurium that carries the therapeutic genes has achieved promising results in animal experiments, including in mouse melanoma, liver cancer, renal cell carcinoma, breast cancer and gastric cancer $(24,25)$. The key problem in RNAi treatment is that RNAi fragments do not reach the effective site and achieve the effective dose. Ji et al confirmed in the mouse prostate model that $S$. typhi is capable of carrying siRNA fragments deep inside the tumor and can inhibit tumor growth and metastasis (24). S. typhi has a high degree of tumor targeting; therefore, it is a good targeting vector for RNA interference.

In this study, we selected attenuated S. typhimurium strain SL7207 that has deleted 5-enol acetone phthalocyanine oxalate-3-phosphate synthase aroA gene as a dual-function plasmid vector in order to evaluate the activity of the dual-function plasmid in vivo by oral gavage treatment of nude mice. SL7207 with the aroA gene defect is only able to proliferate to a limited level in mammalian cells prior to death, and then the bacteria release the expression plasmid inside the bacteria. These plasmids express their encoded protein in the cytoplasm. This bacterial strain has low toxicity but still retains the cell invasive property, and is capable of carrying encoded exogenous gene eukaryotic expression plasmid into the tumor tissue and ultimately achieving therapeutic effects by the gene expression product (26). The results show that SL7207 infects breast cancer cells effectively in vitro and in vivo. Although SL7207 gathered in the normal organs of the liver and spleen within a short time, it is capable of remaining at high concentrations in tumors for long periods of time and can express the targeting products effectively. As the expression of ENDO-VEGI151 recombinant protein and its anti-angiogenic effect could be affected by survivin siRNA-induced tumor cell apoptosis, the two therapeutic genes have a synergistic effect in tumor elimination. However, due to the phase difference of apoptosis, non-apoptotic or not fully apoptotic cells may express ENDO-VEGI151. ENDO-
VEGI151 may be secreted to the extracellular domain due to the presence of IL3 signal peptide. Our immunohistochemical results also suggest that interstitial tumor tissue also has fusion protein expression, therefore we suggest that the expression of the two target genes did not interfere with each other. However, for combination therapy, the appropriate ratio of the two drugs (ENDO-VEGI and survivin small molecule siRNA) is essential for optimizing their synergistic effect and safety, and this should be explored further.

\section{Acknowledgements}

This study was supported by the National Natural Science Foundation of China (no. 51003078), the Shanghai Municipal Health Bureau Research Projects (no. 2008133) and the Shanghai Municipal Science and Technology Commission (No. 12140902302).

\section{References}

1. Jemal A, Siegel R, Xu J and Ward E: Cancer statistics, 2010. CA Cancer J Clin 60: 277-300, 2010.

2. Grammatikakis I, Zervoudis S and Kassanos D: Synopsis of new antiangiogenetic factors, mutation compensation agents, and monoclonal antibodies in target therapies of breast cancer. J BUON 15: 639-646, 2010.

3. Stoff-Khalili MA, Dall P and Curiel DT: Gene therapy for carcinoma of the breast. Cancer Gene Ther 13: 633-647, 2006.

4. Butler JM, Kobayashi $\mathrm{H}$ and Rafii S: Instructive role of the vascular niche in promoting tumour growth and tissue repair by angiocrine factors. Nat Rev Cancer 10: 138-146, 2010.

5. Weigand M, Hantel P, Kreienberg $\mathrm{R}$ and Waltenberger $\mathrm{J}$ : Autocrine vascular endothelial growth factor signalling in breast cancer. Evidence from cell lines and primary breast cancer cultures in vitro. Angiogenesis 8: 197-204, 2005.

6. Li Z, Yang S, Chang T, Cao X, Shi L and Fang G: Antiangiogenesis and anticancer effects of a plasmid expressing both ENDO-VEGI151 and small interfering RNA against survivin. Int J Mol Med 29: 485-490, 2012.

7. Cao S, Cripps A and Wei MQ: New strategies for cancer gene therapy: progress and opportunities. Clin Exp Pharmacol Physiol 37: 108-114, 2010.

8. Jimeno A and Hidalgo M: Multitargeted therapy: can promiscuity be praised in an era of political correctness? Crit Rev Oncol Hematol 59: 150-158, 2006.

9. Zhang Y, Qu ZH, Cui M, Guo C, Zhang XM, Ma CH and Sun WS: Combined endostatin and TRAIL gene transfer suppresses human hepatocellular carcinoma growth and angiogenesis in nude mice. Cancer Biol Ther 8: 466-473, 2009.

10. Yu B, Zhang Y, Zhan Y, Zha X, Wu Y, Zhang X, Dong Q, Kong W and $\mathrm{Yu} X$ : Co-expression of herpes simplex virus thymidine kinase and Escherichia coli nitroreductase by an hTERT-driven adenovirus vector in breast cancer cells results in additive antitumor effects. Oncol Rep 26: 255-264, 2011.

11. Abdollahi A and Folkman J: Evading tumor evasion: current concepts and perspectives of anti-angiogenic cancer therapy. Drug Resist Updat 13: 16-28, 2010.

12. Miles DW, Chan A, Dirix LY, et al: Phase III study of bevacizumab plus docetaxel compared with placebo plus docetaxel for the first-line treatment of human epidermal growth factor receptor 2-negative metastatic breast cancer. J Clin Oncol 28: 3239-3247, 2010.

13. Robert NJ, Diéras V, Glaspy J, et al: RIBBON-1: randomized, double-blind, placebo-controlled, phase III trial of chemotherapy with or without bevacizumab for first-line treatment of human epidermal growth factor receptor 2-negative, locally recurrent or metastatic breast cancer. J Clin Oncol 29: 1252-1260, 2011.

14. Kerbel RS: Issues regarding improving the impact of antiangiogenic drugs for the treatment of breast cancer. Breast 18 (Suppl 3): S41-S47, 2009.

15. Leite de Oliveira R, Hamm A and Mazzone M: Growing tumor vessels: more than one way to skin a cat -implications for angiogenesis targeted cancer therapies. Mol Aspects Med 32: 71-87, 2011. 
16. Pang RW and Poon RT: Clinical implications of angiogenesis in cancers. Vasc Health Risk Manag 2: 97-108, 2006.

17. Eichhorn ME, Kleespies A, Angele MK, Jauch KW and Bruns CJ: Angiogenesis in cancer: molecular mechanisms, clinical impact. Langenbecks Arch Surg 392: 371-379, 2007.

18. Carrasco RA, Stamm NB, Marcusson E, Sandusky G, Iversen P and Patel BK: Antisense inhibition of survivin expression as a cancer therapeutic. Mol Cancer Ther 10: 221-232, 2011.

19. Rice $\mathrm{C}$ and Huang LE: From antiangiogenesis to hypoxia: current research and future directions. Cancer Manag Res 3: 9-16, 2010.

20. Peng XH, Karna P, Cao Z, Jiang BH, Zhou M and Yang L: Cross-talk between epidermal growth factor receptor and hypoxia-inducible factor-1alpha signal pathways increases resistance to apoptosis by up-regulating survivin gene expression. J Biol Chem 281: 25903-25914, 2006.

21. Liu C, Liang B, Wang Q, Wu J and Zou MH: Activation of AMP-activated protein kinase alpha1 alleviates endothelial cell apoptosis by increasing the expression of anti-apoptotic proteins Bcl-2 and survivin. J Biol Chem 285: 15346-15355, 2010.
22. Gardlik R and Fruehauf JH: Bacterial vectors and delivery systems in cancer therapy. IDrugs 13: 701-706, 2010.

23. Gardlik R, Behuliak M, Palffy R, Celec P and Li CJ: Gene therapy for cancer: bacteria-mediated anti-angiogenesis therapy. Gene Ther 18: 425-431, 2011.

24. Ji K, Wang B, Shao YT, et al: Synergistic suppression of prostatic cancer cells by coexpression of both murine double minute 2 small interfering RNA and wild-type p53 gene in vitro and in vivo. J Pharmacol Exp Ther 338: 173-183, 2011.

25. Zhu X, Zhou P, Cai J, Yang G, Liang S and Ren D: Tumor antigen delivered by Salmonella III secretion protein fused with heat shock protein 70 induces protection and eradication against murine melanoma. Cancer Sci 101: 2621-2628, 2010.

26. Lu XL, Jiang XB, Liu RE and Zhang SM: The enhanced antiangiogenic and antitumor effects of combining flk1-based DNA vaccine and IP-10. Vaccine 26: 5352-5357, 2008. 